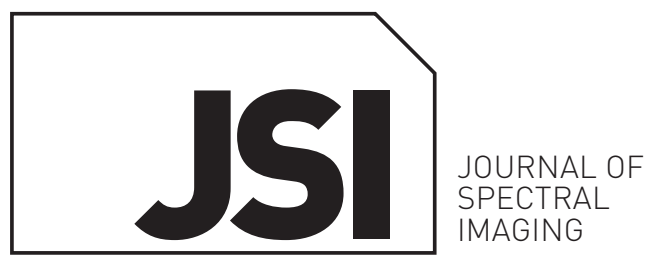

\title{
Use of spectral pre-processing methods to compensate for the presence of packaging film in visible-near infrared hyperspectral images of food products
}

\author{
A.A. Gowen, ${ }^{*}$ G. Downey, C. Esquerre and C.P. O’Donnell \\ Biosystems Engineering, School of Agriculture, Food Science and Veterinary Medicine, University College Dublin, Belfield, Dublin 4, Ireland. \\ E-mail: aoife.gowen@ucd.ie
}

\begin{abstract}
The presence of polymeric packaging film in images of food products may modify spectra obtained in hyperspectral imaging (HSI) experiments, leading to undesirable image artefacts which may impede image classification. Some pre-processing of the image is typically required to reduce the presence of such artefacts. The objective of this research was to investigate the use of spectral pre-processing techniques to compensate for the presence of packaging film in hyperspectral images obtained in the visible-near infrared wavelength range $(445-945 \mathrm{~nm})$, with application in food quality assessment. A selection of commonly used pre-processing methods, used individually and in combination, were applied to hyperspectral images of flat homogeneous samples, imaged in the presence and absence of different packaging films (polyvinyl chloride and polyethylene terephthalate). Effects of the selected pre-treatments on variation due to the film's presence were examined in principal components score space. The results show that the combination of first derivative Savitzky-Golay followed by standard normal variate transformation was useful in reducing variations in spectral response caused by the presence of packaging film. Compared to other methods examined, this combination has the benefits of being computationally fast and not requiring a priori knowledge about the sample or film used.
\end{abstract}

Keywords: hyperspectral, imaging, pre-processing, packaging, film, polymer, food

\section{Introduction}

Packaging of foods extends their shelf-life by decelerating deteriorative changes, such as spoilage and dehydration, arising from environmental conditions and offering protection from contamination. Polymeric films such as polyvinyl chloride (PVC) and polyethylene terephthalateand (PET) are commonly used in post-harvest packaging of fruit and vegetables. ${ }^{1}$ Numerous applications of hyperspectral imaging (HSI) have been presented for quality evaluation of food and pharmaceutical products. ${ }^{2-4}$ Hyperspectral imaging of food products through packaging would be useful for post-harvest quality evaluation and verification, eliminating the need to remove such products from packaging prior to testing. In addition, performing laboratory-based HSI experiments on packaged food products would reduce the effect of the ambient environment on the sample's properties.

In a recent paper, the authors investigated the spectral and spatial effects that may be encountered when acquiring hyperspectral images of objects covered in polymeric film. ${ }^{5}$ Typical polymer films used for food packaging transmit approximately $90 \%$ of light in the visible-near infrared [vis-NIR (400-1000 nm)] wavelength range. Although such films do not exhibit strong absorbance features in this wavelength range, changes in the optical path length of light transmitted through the film leads to interference fringes ${ }^{6}$ that may distort spectral 
data obtained from both point spectroscopy and hyperspectral imaging experiments. Transmitted light becomes scattered by multiple reflections at the air-film-air interfaces. Typically, a portion of light will be specularly reflected from the film surface and this combines with light diffusely reflected from the sample, which may reduce the overall integrity of information contained in the hyperspectral image. The effect of film orientation on scattering is considerable and this may not always be well defined in most practical situations. Therefore, there is a need for pre-processing methods applicable to HSI data that can overcome the scattering effects arising when objects are imaged through film.

Chemometric techniques for analysis of multivariate data such as principal components analysis (PCA) and partial least squares (PLS) are often combined with spectral preprocessing methods such as standard normal variate (SNV), multiplicative scatter correction (MSC), detrending and derivative pre-treatment. These pre-processing methods are frequently used in point spectroscopy to reduce scattering effects. ${ }^{7}$ Multivariate image analysis (MIA) extends chemometric techniques, developed originally for the analysis of point spectra, to multivariate imaging data including multispectral ${ }^{8-11}$ and hyperspectral ${ }^{4,12}$ images. Hyperspectral imaging can, in turn, offer new insights into chemometric techniques. Fearn et. al. ${ }^{13}$ recently demonstrated that the pretreatments SNV and MSC behave very differently to each other when applied to HSI data; something that was not immediately obvious when considering point spectra. ${ }^{14}$ Through analysis of the geometry of each pre-treatment on simulated data, they showed that SNV tends to induce curved structures in PC scores space while MSC tends to result in outliers. The aim of this paper is to explore the use of spectral pre-processing methods for compensation of the effects encountered when acquiring hyperspectral images of samples covered in polymeric packaging film.

\section{Materials and methods Packaging films}

PVC packaging film of $18 \pm 1 \mu \mathrm{m}$ thickness ("Lin-Wrap" film, Linpac Plastics Ltd, Birmingham, UK) and PET packaging film of $30 \pm 1 \mu \mathrm{m}$ thickness (Dupont Plastics Ltd, Stevenage, UK) was used in all experiments. These films are typically used for packaging of foods (for example, fruit, vegetables and frozen products) and are also commonly used in packaging of pharmaceutical products.

\section{Samples studied}

To examine the effect of polymer films on hyperspectral imaging data, a selection of samples were tested as described below:

1. to represent a flat, uniform, highly-reflecting sample, a white tile (Ceram Research Ltd, Stoke-on-Trent, UK) was used;

2. to represent samples absorbing light in the visible wavelength range, red and green paper samples cut from paint colour reference sheets were used (Fleetwood Ltd, Virginia, Co. Cavan, Ireland, product code: AC 48, AC 88);

3. to represent food products with spectral response in the vis-NIR wavelength region, mushroom samples [Agaricus bisporus spp. grown in Teagasc Research Centre (Kinsealy, Co. Dublin, Ireland)] and a pear (purchased in a local supermarket) were employed. The former was packaged in PVC and the latter in PET film.

\section{Hyperspectral image acquisition}

The hyperspectral imaging system (Spectral Scanner, DV Optics, Padua, Italyl used in this research consisted of a high performance CCD camera $(580 \times 580$ pixels), spectrograph (Specim V10E, Spectral Imaging Ltd., Oulu, Finland) attached to the camera covering the spectral range between $400 \mathrm{~nm}$ and $1000 \mathrm{~nm}$, a zoom lens, tungsten halogen light source transmitted through a fibre-optic line light covered with a diffusing column, moving translation stage and a computer to acquire the images. ${ }^{5}$ Only spectral data in the $445-945 \mathrm{~nm}$ wavelength region was used in the analysis due to low efficiency of the light source and CCD beyond this region.

A two-point reflectance calibration was performed on the HSI data; the bright response ("W") was obtained by collecting a hypercube from a uniform white ceramic, the reflectance of which was calibrated against a tile of certified reflectance (Ceram Research Ltd, UK); the dark response ("dark") was acquired by turning off the light source, completely covering the lens with its cap and recording the camera response. The corrected reflectance value $(R)$ was calculated from the measured signal ("I") on a pixel-by-pixel basis as shown below:

$$
R i=(l i-\text { dark }) /(\text { Wi-dark })
$$

where $i$ is the pixel index, i.e. $i=1,2,3, \ldots, n$ and $n$ is the total number of pixels.

Mean reflectance spectra of each sample studied were obtained from hyperspectral images by interactively selecting rectangular regions (containing $\sim 100 \times 100$ pixels) from the mean image of each sample.

In order to approximate a flat film surface and to avoid variations due to changes in sample position between scans, a sheet of each film $30 \times 50 \mathrm{~cm}$ in size was attached to a cardboard frame. Hyperspectral images of each sample were first obtained without the film; immediately afterwards, the sample was scanned underneath the film. The film was placed above the sample (with approximately $1 \mathrm{~cm}$ gap of air between the sample and film) on the translation stage so that it moved along the scanning direction with the sample.

\section{Data analysis}

All image and spectroscopic analyses were carried out in Matlab (Version 7, The MathWorks Inc., Massachusetts, USA). 
False colour RGB images were obtained by concatenating image planes at $460 \mathrm{~nm}$ (blue), $550 \mathrm{~nm}$ (green) and $650 \mathrm{~nm}$ (red). Mean hyperspectral images were obtained by averaging the pixel response over the wavelength range. Data obtained from hyperspectral images were subjected to common spectral pre-processing methods as described in Table 1. PCA can be applied to spectral data to form linear combinations of the individual wavelengths in directions of maximal variance. As such, PCA is a useful tool for identifying clusters in multidimensional data. PCA was applied to the hyperspectral data after unfolding (from a three-dimensional hypercube structure to a two-dimensional matrix of spectral and PC eigenvectors or loadings were applied to hyperspectral images to construct PC score images. ${ }^{10}$ PC score "difference" images were obtained by subtracting PC scores of samples imaged in the presence and absence of film. Unless otherwise indicated, PCA was applied to data from each image separately.

\section{Results and discussion}

Relatively homogeneous and flat samples (white tile, red and green paper) were initially examined in order to investigate the effects of the selected pre-treatments. False colour images of the flat samples, imaged in the absence and presence of each film, are shown in Figure 1(a). Due to reflection of light from the surface of the film, the objects covered in PVC appeared lighter in colour compared to those imaged without the film. The PET film clearly had a greater effect on the image than the PVC; surface topography of the film resulted in regions of image lightening and darkening. Histograms of the mean hyperspectral image of each sample are also shown [Figure $1(b)]$. In the case of the image taken in the absence of film, four distinct peaks are evident in the histogram, representing each object present in the image (i.e. background, white tile, red paper, green paper). Shifts to higher intensity pixel values, accompanied by changes in histogram shape, are evident in the histograms of the images taken in the presence of each film, with a substantially greater shift occurring for the PET than for the PVC film. This is due to additional scattering of light from the film surface back towards the detector.

Considering the mean spectrum of each region [plotted in Figure $1(c)$, it can be seen that the films resulted in increased reflectance values for each object at all wavelengths and the effect of the film was different for the different objects examined; the amount of specular reflection was relatively high for the red paper covered in PET film [see Figure 1 (a)]; this may be related to its thickness laround 30\% thicker than

(a)

(i)

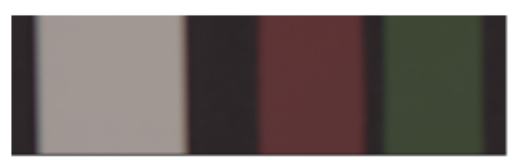

(ii)

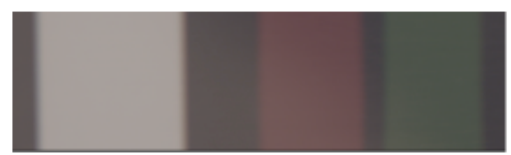

(iii)

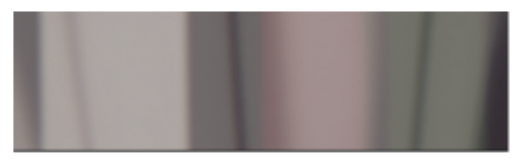

(c)

(i)

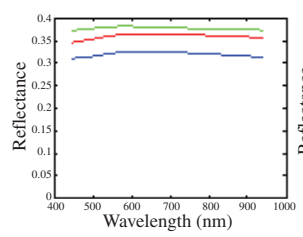

(ii)

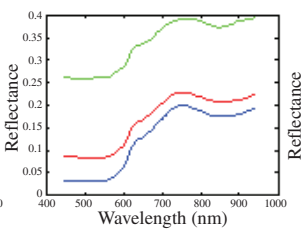

(iii)

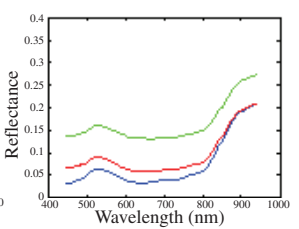

(b)

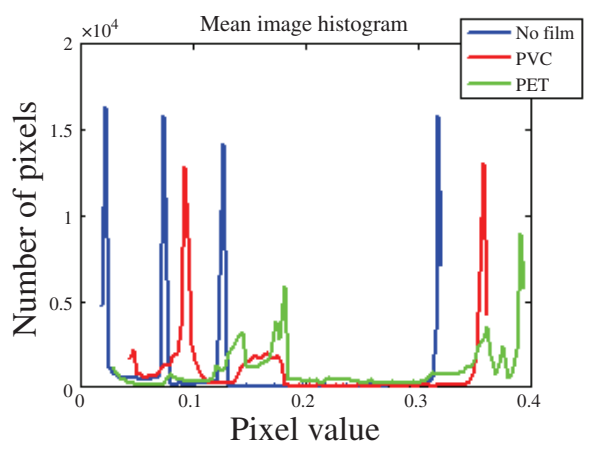

(d)

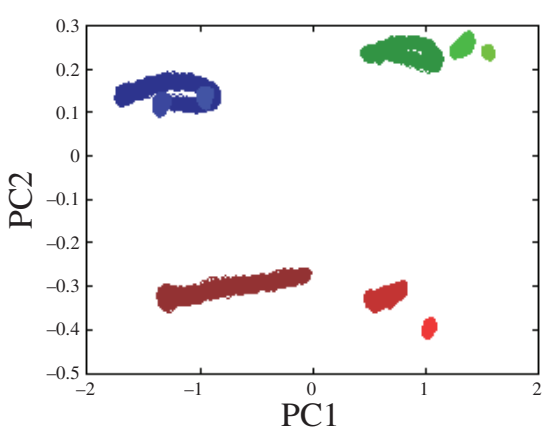

Figure 1. (a) False colour RGB images of white tile, red and green paper in (i) the absence of packaging film, (ii) the presence of PVC packaging film, (iii) the presence of PET packaging film; (b) mean hyperspectral image histograms for samples shown in (a), where the blue line =image taken in the absence of film, the red line=image taken in the presence of PVC film, green line=image taken in the presence of PET film; (c) mean spectra of (i) white tile, (ii) red paper and (iii) green paper imaged in the absence of film (blue line), in the presence of PVC film (red line) and in the presence of PET film (green line); (d) PC1 and PC2 scores for all spectra analysed together, where blue = white tile, red = red paper and green = green paper; colour intensity is related to the presence/absence of packaging film where the brightest intensity relates to its absence and the darkest to the presence of PET film. 
Table 1. Description of spectral pre-processing methods employed.

\begin{tabular}{|c|c|}
\hline Abbreviation & Description \\
\hline SNV & Standard normal variate ${ }^{12,14}$ \\
\hline MSC & Multiplicative scatter correction ${ }^{12,13}$ \\
\hline EMSC & Extended multiplicative scatter correction ${ }^{15}$ \\
\hline SG1 & $1^{\text {st }}$ Derivative Savitsky-Golay (window size $=15$, polynomial order $\left.=3\right)^{11}$ \\
\hline $\mathrm{D}$ & Detrending $\left(2^{\text {nd }} \text { order polynomial }\right)^{16,17}$ \\
\hline SG2 & $2^{\text {nd }}$ Derivative Savitsky-Golay (window size $=15$, polynomial order $=3$ ) ) $^{11}$ \\
\hline SG1+SNV & SG1 followed by SNV \\
\hline SNV + SG1 & SNV followed by SG1 \\
\hline $\mathrm{D}+\mathrm{SNV}$ & D followed by SNV \\
\hline$S N V+D$ & SNV followed by D \\
\hline$S G 1+D$ & SG1 followed by D \\
\hline $\mathrm{D}+\mathrm{SG1}$ & D followed by SG1 \\
\hline $\mathrm{D}+\mathrm{SG1+SNV}$ & D followed by SG1 followed by SNV \\
\hline SG1+D+SNV & SG1 followed by D followed by SNV \\
\hline $\mathrm{D}+\mathrm{SNV}+\mathrm{SG} 1$ & D followed by SNV followed by SG1 \\
\hline
\end{tabular}

the PVCl and also due to the non-flatness of the PET film leading to multiple surface reflections. ${ }^{4}$

In order to further investigate the effect of the films on the acquired images, the spectra from each region and film presence/absence combination were combined into a single matrix on which PCA was applied. The resultant principal component $(P C)$ scores are shown in Figure 1(d). Each object (i.e. white tile, red paper, green paper) is clearly separated in PC1-PC2 scores space and, within each object, the different imaging conditions examined (i.e. no film, PVC film, PET film) are clearly distinguishable. Noticeably, the spread of pixel values in PC score space is increased in the presence of the film. This may be related to surface scattering of light by the film; the PET film had the greatest effect on the pixel spectra in this respect. Different spectral pre-treatments (described in Table 1) were applied to the data, prior to performing PCA, in order to explore their effects on pixel distributions in PC score space (Figure 2).

All pre-treatments studied resulted in a decrease in the percentage variance explained by the first two PCs from $99 \%$ (when no pre-treatment was applied) to $60-80 \%$. This is a common feature of hyperspectral data in which the majority of variability, usually representing multiplicative effects and baseline shifts originating from variability in light intensity, calibration and sample morphology, is extracted in the first PC. Application of spectral pre-treatments decreases or removes such variability. The objective of applying the selected pre-treatments was to decrease the variability in the data caused by the presence of the film while preserving the variability due to inherent sample differences. In PC scores space, this amounts to maintaining the distance between clusters of pixels belonging to different objects while at the same time decreasing the spread between clusters of the same object due to the film. SNV [Figure 3(a)] performed well in this respect for the red and green paper but did not effectively decrease film-related variability for the white tile. Due to the flat spectrum of the white tile (Figure 1), the standard deviation of each spectrum is small compared to the other materials; this results in a greater spread in $\mathrm{PC}$ score space after SNV pre-treatment. Both MSC and extended multiplicative scatter correction (EMSC) resulted in large outliers in PC scores space; the tendency of MSC to produce such effects is well described by Fearn et al. ${ }^{13}$ The outliers correspond to spectra that lie nearly orthogonal to the reference spectrum used in MSC, resulting in a slope near to zero.

The SG1 pre-treatment decreased film-related variability for the white tile but was less effective at doing so for the red and green paper. Application of SG1 followed by SNV appeared to be a promising pre-treatment, decreasing the variability due to the film while maintaining object-to-object variability. However, this combination of pre-treaments resulted in some distortion in the shape of pixel clusters observed in PC score space. The application of SG1 alone resulted in narrow, vertically inclined clusters; SNV alone resulted in more curved cluster shapes. The curved cluster shapes induced by SNV are expected. according to the geometric treatment of the behaviour of SNV in PC scores space given by Fearn et al. ${ }^{13}$ The combination of SG1 followed by SNV resulted in ellipsoidal cluster shapes. The induced distortion in pixel distribution may affect classification methods (for example, Mahalanobis distance) that assume normal distribution of scores. ${ }^{13}$ Changing the order of the pre-treatments (i.e. SNV followed by SG1) resulted in overlapping clusters; this combination decreased the variability due to the film but also removed the variability due to inherent sample differences, which is undesirable. The application of SG2 had a similar effect.

Detrending seemed to have a similar effect to SG1, decreasing film-related variability for the white tile but not for the red and 

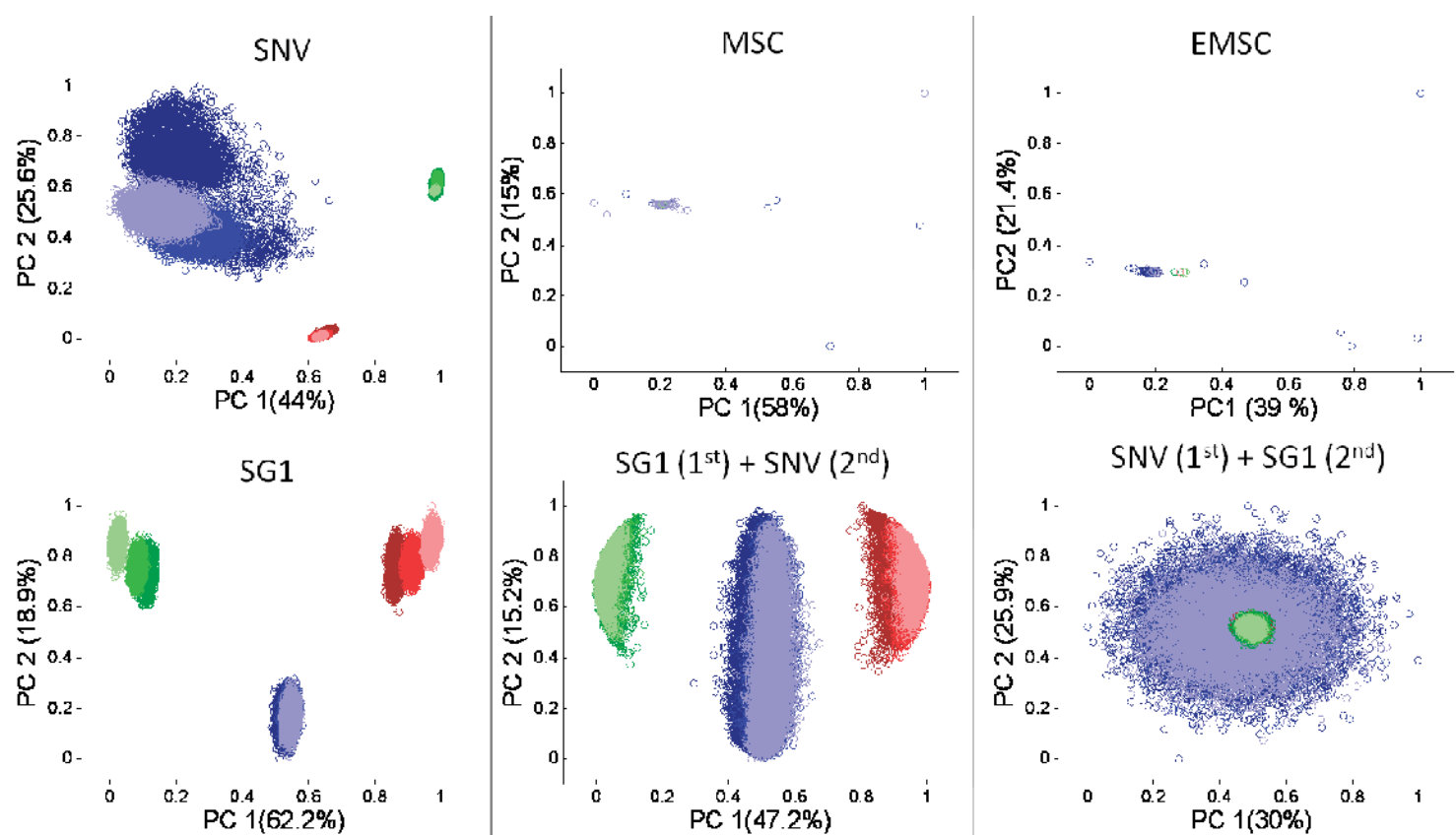

SNV $\left(1^{\text {st }}\right)+\operatorname{SG} 1\left(2^{\text {nd }}\right)$

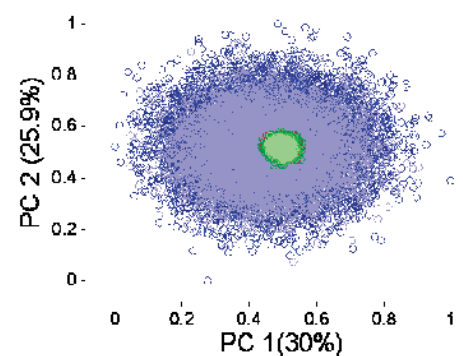

D
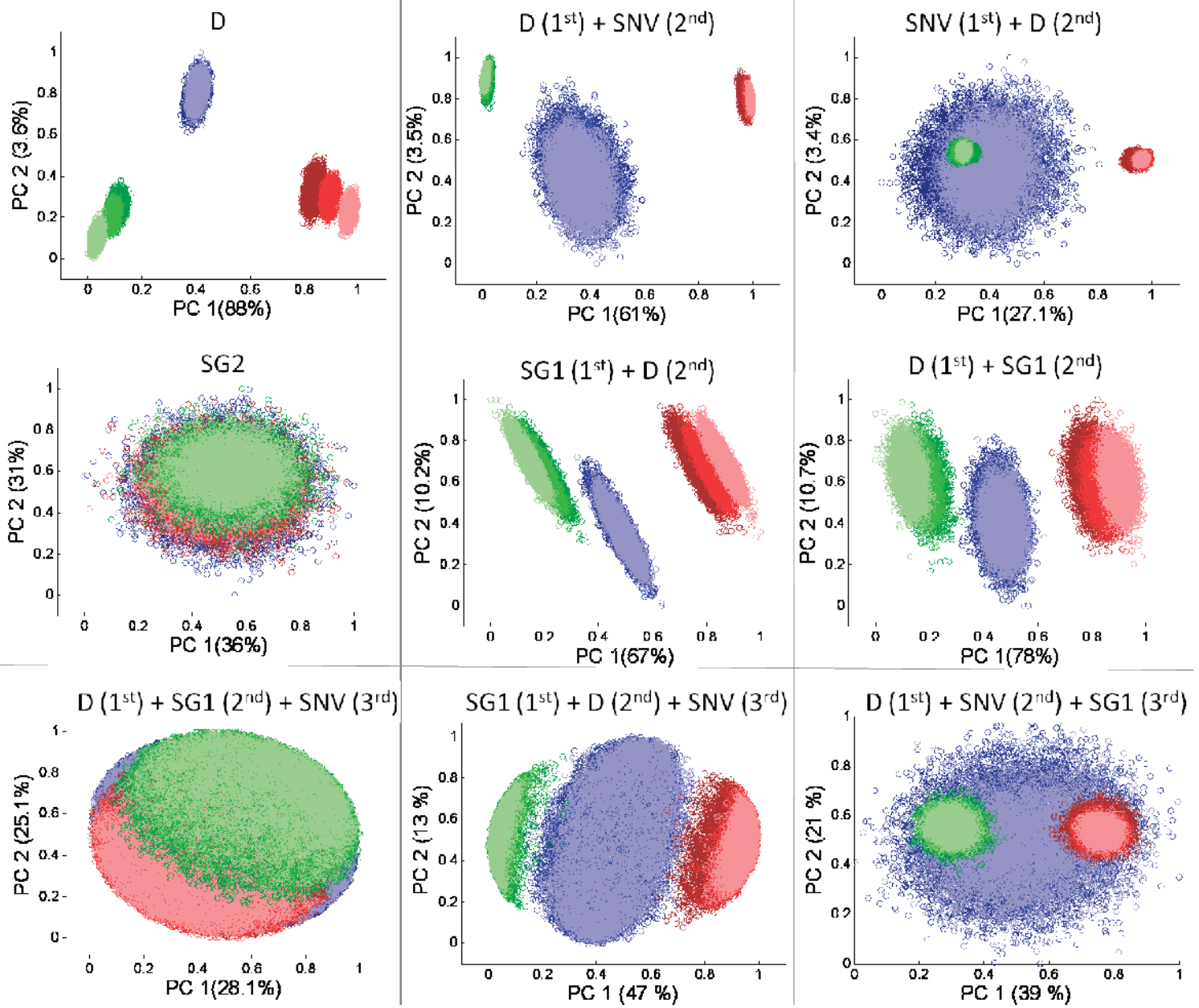

Figure 2. PC1 and PC2 scores for spectra of white tile, green paper and red paper (analysed together) after applying different spectral pre-processing methods (described in Table 1) where blue = white tile, red = red paper and green = green paper; colour intensity is related to the presence/absence of packaging film where the brightest intensity relates to its absence and the darkest to the presence of PET film. 
green paper. Detrend (D) followed by SNV was another promising combination, decreasing variability due to the films while maintaining object-to-object variability. Reversing the order of this combination resulted in overlapping of the clusters representing the green and white samples. SG1 followed by D and the reverse order combination of these two pre-treatments also performed relatively well, resulting in some overlap in pixels of the same object imaged in different films while also maintaining objectto-object variability. The three best performing pre-treatments (D, SG1 and SNV) were also combined in different orders and applied to the data. However, although these combinations decreased the variability due to the films, they generally led to reduced separation of the different objects in score space and will not, therefore, be discussed further here.

Based on the preceding visual analysis of PC score plots, candidates for suitable pre-processing methods meriting further investigation were selected as: SG1, SG1+SNV, D+SG1 and D+SNV. Although the score plots shown in Figure 2 were helpful in selecting candidate pre-treatments, they do not allow visualisation of pixel density within the clusters. The use of histograms to visualise PC scores is preferable in this respect, enabling improved visualisation of the distribution of pixel values for a particular PC. Histograms of PC1-3 scores for each candidate pre-treatment are shown in Figure 3. All

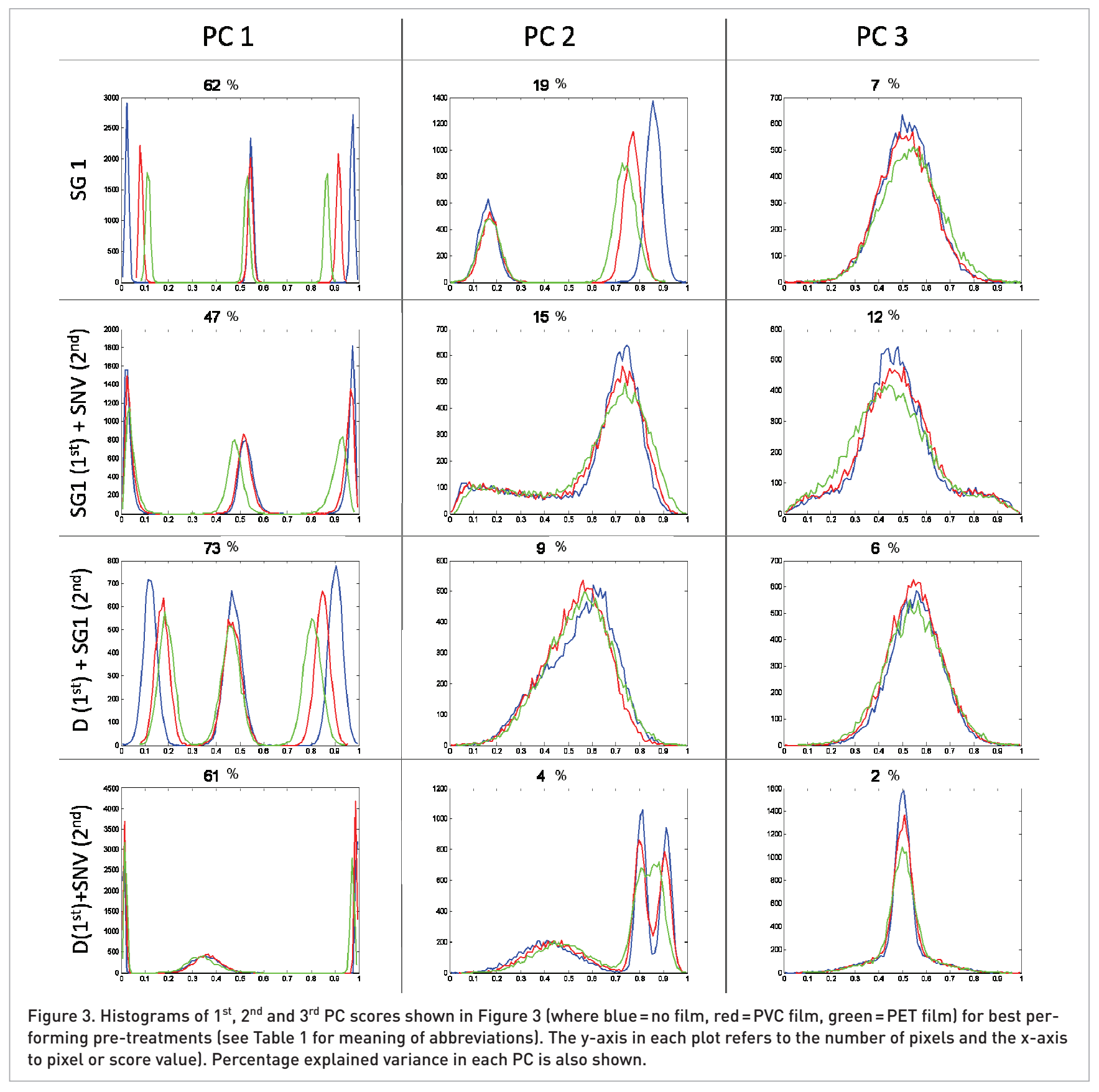


pre-treatments performed well on PC3, leading to overlapping histograms, indicating similar distribution of pixels in the PC3 score space in the absence or presence of film.
Considering PC1 and PC2 scores, SG1+SNV and D+SNV performed better than the other two candidates, leading to more overlapping of histograms.

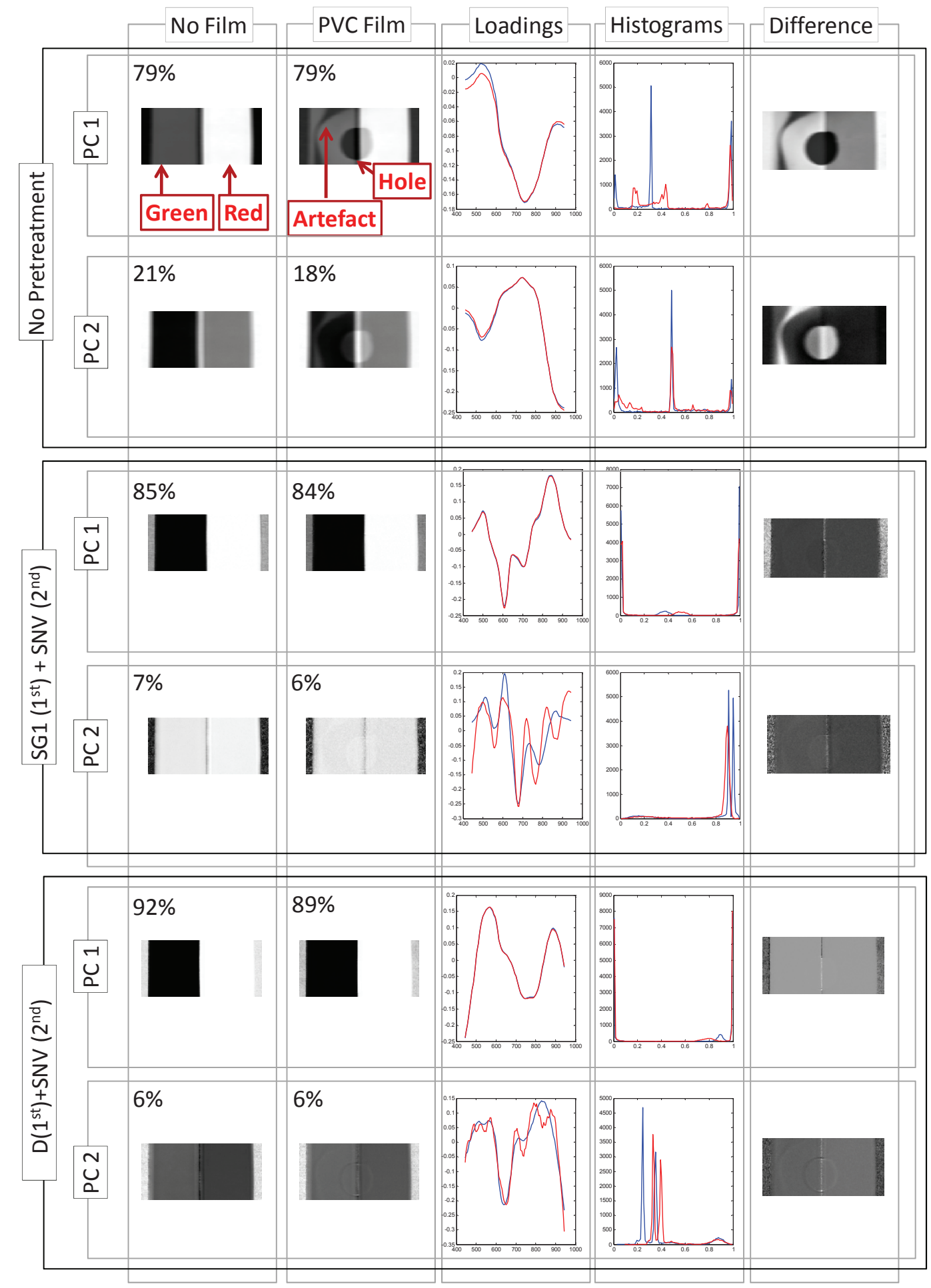

Figure 4. Red and green paper imaged in the absence and presence of PET film with a hole in the centre. PC1 and PC2 score images of non-pre-treated, first derivative Savitzky-Golay followed by standard normal variate (SG1+SNV) and detrending followed by SNV (D+SNV) pre-treated data. Percentage variance of each PC, loadings, PC score histograms and difference images (film-no film) are also shown. The blue line refers to "no film" and the red line to "film". 
In order to further test the performance of the SG1+SNV and D+SNV pre-treatments, two further images of the red and green paper were obtained: one in the absence of film and another in the presence of PET film but, this time, the film contained a hole in its centre (Figure 4). The hole was intentionally placed so that it covered portions of both the red and green paper. Should the proposed pre-treatments compensate for the film's presence, the hole should not be evident in subsequent PC scores images. In the presence of the film, a line is visible on the right-hand side of the green paper. This may appear, at first glance, to be a tear in the film; however, it is an image artefact caused by a slight curve in the film. PCA was applied to each image individually. The PC loadings for the non-pre-treated data are similar for the sample imaged with and without film. However, due to the spectral differences between the film and non-covered samples, their PC scores are very different, as evidenced in the histograms and PC score images shown. Score images for the non-pre-treated data reveal numerous image artefacts in the film-covered sample. These artefacts are more clearly evident in the difference images (also shown in Figure 4) and make the flat paper seem highly inhomogenous due to surface reflection where the film is present.
After application of the SG1+SNV pre-treatment, the score images for the samples imaged in the absence of film and in its presence became nearly identical to each other and artefacts due to the film's presence are hardly visible. Histograms of the score images confirm this. The hole is not evident in the film-covered sample. However, the loadings of the $2^{\text {nd }} P C$ are not identical for film- and non-covered samples. The difference images exhibit some residual evidence of the film; however, this is very slight compared with that of the non-pre-treated image. The D+SNV pre-treatment was also successful in decreasing the effect of the film on this sample. However, the $\mathrm{D}+\mathrm{SNV}$ pre-treatment required more computational time $140 \mathrm{~s}$ for 20,000 pixel spectra) compared to SG1+SNV (4s for 20,000 pixel spectra); this may limit its practicability for processing larger amounts of data. For this reason, only the SG1+SNV pre-treatment is considered in the following sections.

In order to further investigate the appropriateness of the selected pre-treatment (SG1+SNV), hyperspectral images of food samples were obtained in the absence and presence of film. Mushrooms were imaged in PVC film and the pear was imaged in PET film. PCA was performed separately on each image, with and without SNV and SG1 pre-treatment. Principal component score images of the samples are shown in Figures 5 and 6 ;
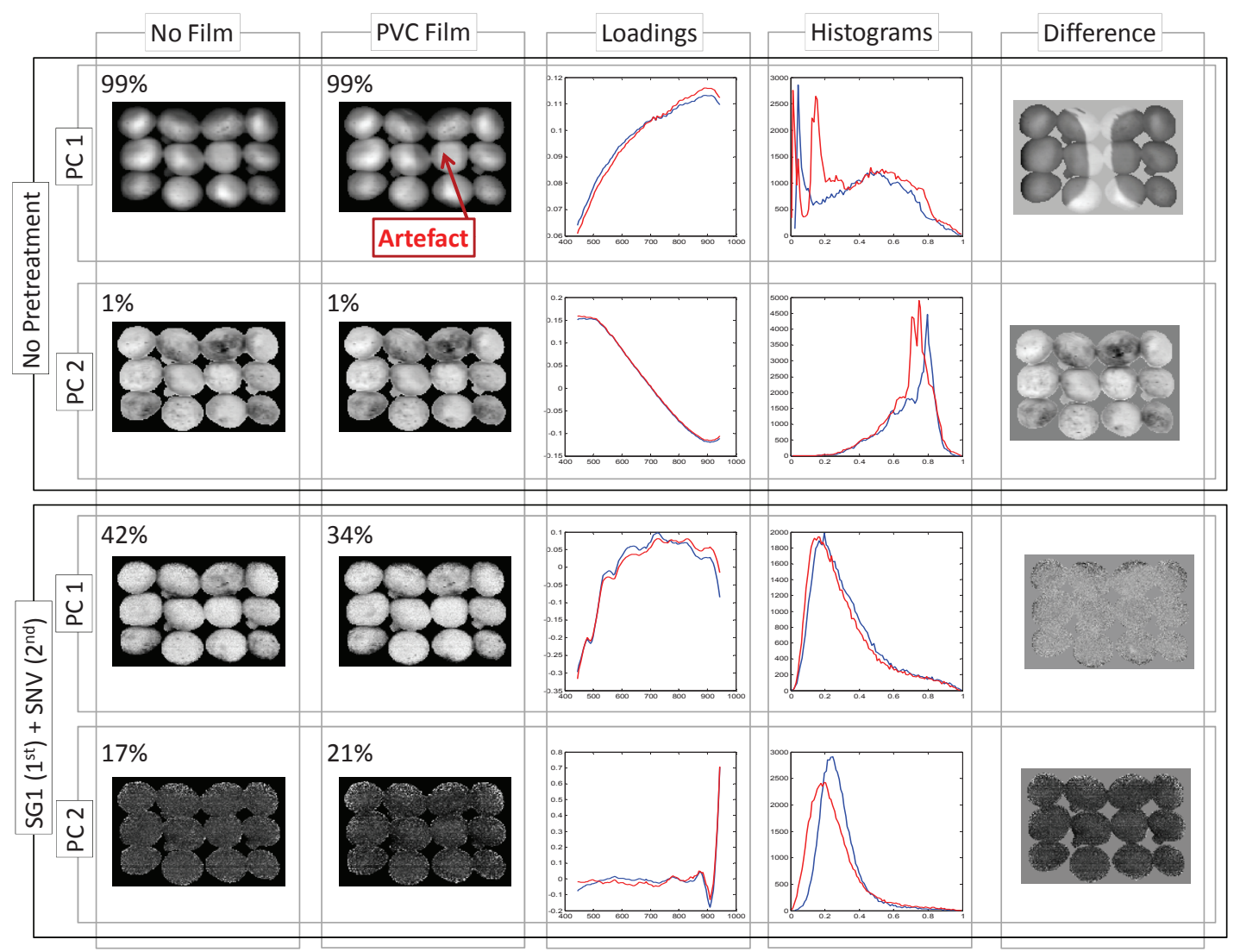

Figure 5. Mushrooms imaged in the absence and presence of PVC film. PC1 and PC2 score images of non-pre-treated and first derivative Savitzky-Golay followed by standard normal variate $(S G 1+S N V)$ pre-treated data. Percentage variance of each PC, loadings, PC score histograms and difference images (film-no film) are also shown. The blue line refers to "no film" and the red line to "film". 

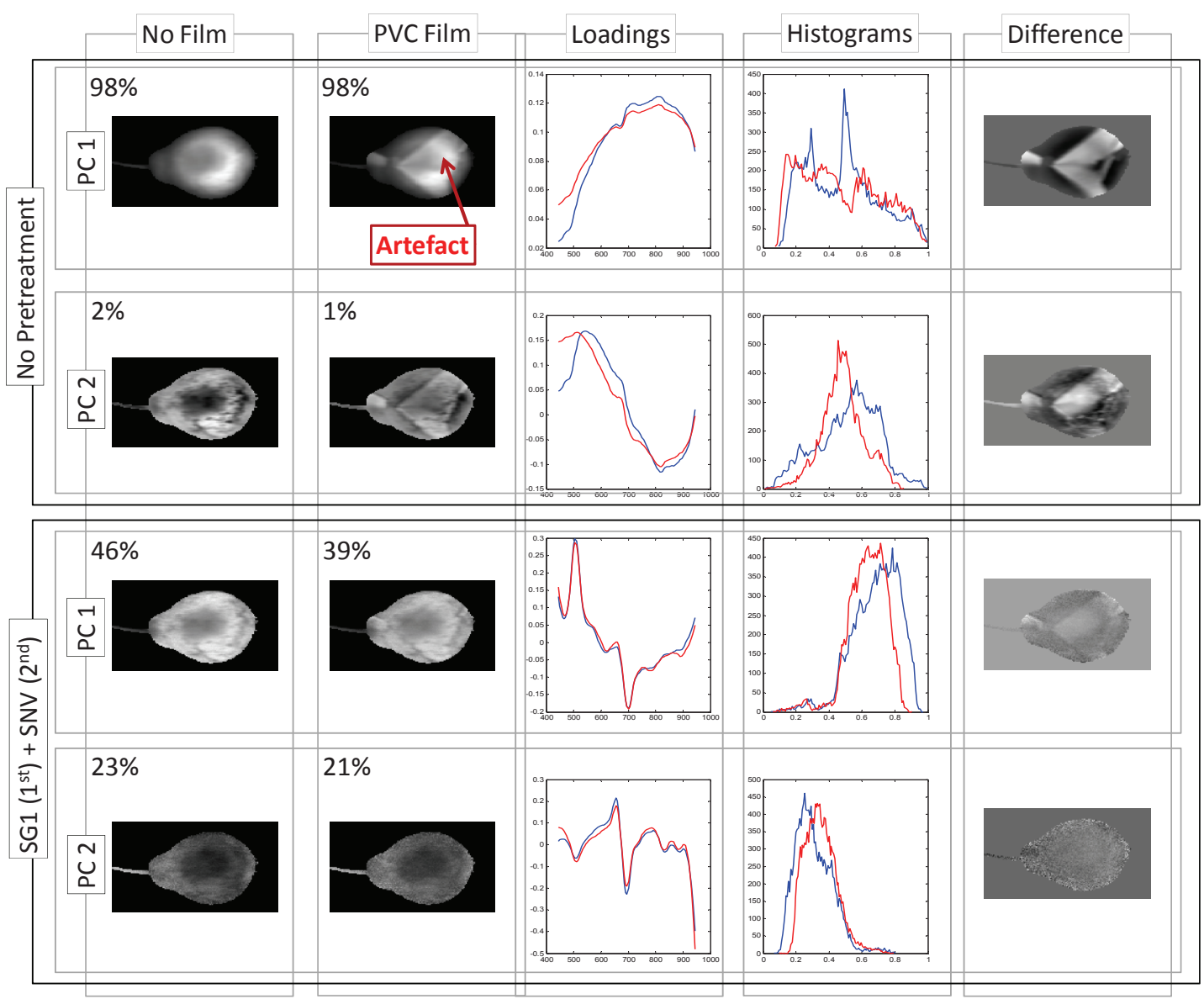

Figure 6. Pear imaged in the absence and presence of PET film. Principal PC1 and PC2 score images of non-pre-treated and first derivative Savitzky-Golay followed by standard normal variate (SG1+SNV) pre-treated data. Percentage variance of each PC, loadings, PC score histograms and difference images (film-no film) are also shown. The blue line refers to "no film" and the red line to "film".

regions of surface damage on each sample can be observed as dark brown regions in the PC images of the samples imaged without film. The non-homogenous effect of each film over the sample surface is evident in the score images of the non-pretreated data and image artefacts introduced by the films are clearly evident in the PC difference images.

In the case of mushrooms, the PC loadings for samples imaged in film were similar to those imaged without film. However, similar to the previous example (Figure 4), the resulting score images were very different. This is evidenced in the score images, histograms and difference images. The PC1 difference image shows an artefact along the centre of the mushroom samples, caused by the presence of the film. The PC2 image, however, does not seem to be affected by this artefact. After application of SG1+SNV pre-treatment, PC loadings became similar, though not identical, in shape for the film- and non-covered samples. The PC score images for filmand non-covered samples are more similar to each other than in the absence of pre-treatment, as is evidenced in the histograms and difference images. Again, some small residual effects of the film are visible in the difference image of the PC1 score image, but this is minor compared with the arte- fact present in the non-pre-treated PC1 image of the sample imaged in the film.

The PET film had a far greater effect on the image of the pear than did the PVC film on the image of the mushrooms. This could be related to the greater thickness of the PET film and its surface topography, as previously observed (Figure 1). The effect of the film is clear in the PC loading plots, which exhibit larger differences between film- and non-covered samples than in the previous examples given (Figures 4 and 5). The resultant PC1 and PC2 score images for the non-pre-treated data indicate the presence of a bruise in the centre of the pear, most clearly evident in the PC2 image of the non-covered pear (Figure 6). This bruise is almost completely masked by an artefact caused by the presence of the film. This artefact is visible in the PC1 and PC2 images of the film-covered sample when no pre-treatment was applied. The PC score images of the film and non-covered pear, obtained after applying the SG1+SNV pre-treatment, are very similar to each other and the bruised region on the pear surface is no longer obscured by the film-induced artefact; it is easily identifiable as dark regions on the PC1 and PC2 images. Application of the SG1+SNV pre-treatment also seemed to reduce the effect of sample curvature in the PC score images, 
which may be useful for preventing misclassification of darker edge regions in images. Loading curves for film- and noncovered samples became more similar to each other after the SG1+SNV pre-treatment and the PC score image histograms also became more similar. The difference images showed some residual evidence of the film in $\mathrm{PC1}$; however, this was not the case for PC2.

\section{Conclusions}

Spectral pre-treatments can be applied to reduce the effect of film on the detected response in hyperspectral images of samples covered in polymer packaging film and their performance can be examined in PC score space. A combination of commonly used spectral pre-processing methods, i.e. SG1+SNV and D+SNV, decreased the effect of the film on hyperspectral imaging data, producing similar score space distributions for pixel spectra from non-covered and filmcovered samples. Applying such pre-processing methods to hyperspectral images of food samples reduced the presence of artefacts due to the film and had the added benefit of decreasing spectral variability introduced by sample curvature.

\section{References}

1. Z. Berk, "Food packaging", Food Process Engineering and Technology. Academic Press, San Diego, USA (2009). doi: 10.1016/B978-0-12-373660-4.00026-0

2. A.A. Gowen, C.P. O'Donnell, P.J. Cullen, G. Downey and J.M. Frias, "Hyperspectral imaging-an emerging process analytical tool for food quality and safety control", Trends Food Sci. Technol. 18, 590 (2007). doi: 10.1016/j. tifs.2007.06.001

3. A.A. Gowen, C.P. O'Donnell, P.J. Cullen and S.E. Bell, "Recent applications of chemical imaging to pharmaceutical process monitoring and quality control", Eur. J. Pharm. Biopharm. 69, 10 (2008). doi: 10.1016/j. ejpb.2007.10.013

4. H.F. Grahn and P. Geladi, Techniques and Applications of Hyperspectral Image Analysis. John Wiley \& Sons Ltd, Chichester, UK (2007). doi: 10.1002/9780470010884

5. A.A. Gowen, C.P. O'Donnell, C. Esquerre and G. Downey, “Influence of polymer packaging films on hyperspectral imaging data in the visible-near-infrared (450-950 nm) wavelength range", Appl. Spectrosc. 64, 304 (2010). doi: $\underline{10.1366 / 000370210790918337}$

6. B.D. Teolis, M.J. Loeffler, U. Raut, M. Famá and R.A. Baragiola, "Infrared reflectance spectroscopy on thin films: Interference effects", Icarus 190, 1 (2007). doi: 10.1016/j.icarus.2007.03.023

7. B. Osborne, T. Fearn and P.H. Hindle, Practical NIR Spectroscopy with Applications in Food and Beverage Analysis. Longman Scientific and Technical, Harlow, UK (1993).

8. P. Geladi and H. Grahn, Multivariate Image Analysis in Chemistry and Related Areas: Chemometric Image Analysis. John Wiley \& Sons Ltd, Chichester, UK (1996).

9. K. Esbensen and P. Geladi, "Strategy of multivariate image analysis (MIA)", Chemometr. Intell. Lab. Syst. 7, 67 (1989).doi: 10.1016/0169-7439(89)80112-1

10. P. Geladi, K. Esbensen and S. Wold, "Image analysis and chemical information in images”, Anal. Chim. Acta 191, 473 (1986). doi: 10.1016/S0003-2670(00)86335-7

11. M.H. Bharati and J.F. MacGregor, "Multivariate image analysis for real-time. process monitoring and control", Ind. Eng. Chem. Res. 37, 4715 (1998). doi: 10.1021/ ie980334L

12. J. Burger and P. Geladi, "Spectral pre-treatments of hyperspectral near infrared images: analysis of diffuse reflectance scattering", J. Near Infrared Spectrosc. 15, 29 (2007). doi: 10.1255/jnirs.717

13. T. Fearn, C. Riccioli, A. Garrido-Varo and J.E. GuerreroGinel, "On the geometry of SNV and MSC", Chemometr. Intell. Lab. Syst. 96, 22 (2009). doi: 10.1016/j.chemo$\underline{\text { lab.2008.11.006 }}$

14. M. Dhanoa, S. Lister, R. Sanderson and R. Barnes, "The link between multiplicative scatter. correction (MSC) and standard normal variate (SNV) transformations of NIR spectra", J. Near Infrared Spectrosc. 2, 43 (1994). doi: 10.1255/jnirs.30

15. H. Martens, The EMSC toolbox for MATLAB. http://www. models.life.ku.dk/source/emsctoolbox

16. R.J. Barnes, M.S. Dhanoa and S.J. Lister, "Standard normal variate transformation and de-trending of nearinfrared diffuse reflectance spectra", Appl. Spectrosc. 43, 772 (1989)

17. W. Wu, B. Walczak, D.L. Massart, K.A. Prebble and I.R. Last, "Spectral transformation and wavelength selection in near-infrared spectra classification", Anal. Chim. Acta 315, 243 (1995). doi: 10.1016/0003-2670(95)00347-3. 